\title{
Revisión crítica sobre el síndrome del latigazo cervical (II): ¿cuánto tiempo tardará en curar?.
}

\author{
A critical review of whiplash associated disorders (II): \\ how long will they take to cure?.
}

\section{A. Ortega Pérez 1}

\begin{abstract}
RESUMEN
Como las pruebas de imagen son poco apropiadas, el síndrome del latigazo cervical (SLC) ha de diagnosticarse y seguirse mediante la anamnesis y la exploración física del lesionado. La constelación de trastornos puede clasificarse en cinco niveles, indicadores de gravedad, mientras que la frecuencia y la intensidad de la cervicalgia permiten el seguimiento del SLC y establecer el alta clínica. Se propone un cuestionario para recoger los datos más relevantes para el diagnóstico y el pronóstico del enfermo. La previsión de indemnización por los daños permite sospechar que puedan exagerarse las lesiones y muchos artículos refutan la importancia del SLC. Sin embargo, tienen limitaciones metodológicas o científicas apreciables. Según las investigaciones, aproximadamente un $\mathbf{2 0} \%$ de los afectados estará curado en una semana, el $\mathbf{5 0} \%$ en un mes, el $70 \%$ en 6 meses y el $80 \%$ en dos años. Uno de cada seis sufrirá incapacidad laboral parcial o completa seis meses después del traumatismo. Aunque distintos datos biológicos, psíquicos, sociales y del accidente seleccionan a los lesionados con mayor riesgo de cronificación, el modelo biológico de lesión propone que esta depende de la capacidad de recuperación del órgano lesionado. Un esguince muscular podrá recuperarse en semanas o pocos meses, pero la lesión de los discos, las articulaciones interapofisarias, las cápsulas articulares, etc. seguramente causarán dolor e incapacidad crónicos. La revisión termina comentando algunos aspectos de la terapia relacionados directamente con la evaluación forense de las lesiones.
\end{abstract}

Palabras clave: Diagnóstico clínico, modelo biológico de lesión, pronóstico, revisión, simulación, síndrome del latigazo cervical, tratamiento.

\section{ABSTRACT}

Since imaging tests are not appropriate, whiplash associated disorders (WAD) need to be diagnosed and followed up using anamnesis and physical examination of the patient. Signs and symptoms of lesion can be grouped into five severity category levels, while monitoring the frequency and intensity of neck pain may establish the moment of recovery from WAD. A questionnaire is proposed to collate the data relevant for diagnosis and prognosis. Several papers dispute the importance of WAD, because compensation for suffering lends to the suspicion of exaggeration of symptoms. However, the methodological or scientific limitations of these papers must be taken into account. Roughly, $20 \%$ of patients will be cured within a week, $50 \%$ within a month, $70 \%$ within 6 months and $80 \%$ within 2 years. One in six will have his/her work capacity restricted longer than six months after the accident. Although several biologic, psychiatric, social or accident-related data can identify the patients with a higher risk of persistent WAD, the biologic model of lesion suggests that this depends on the recuperative capacity of the affected neck structure. A muscle sprain can be healed within weeks or months, while lesion in discs, zygapophyseal joints or capsules will, most probably, cause chronic pain and incapacity. This review concludes by discussing some topics related to therapies directly related to expert witness WAD evaluation.

Key words: Biological model of lesion, clinical diagnosis, prognosis, review, simulation, therapy, whiplash associated disorders.

Correspondencia: Dr. Arturo Ortega. Unidad de Medicina Legal. Facultad de Medicina y Ciencias de la Salud. C/ Sant Llorenç, 21. 43201 - Reus (Tarragona). Correo electrónico: aop@fmcs.urv.es.

1 Doctor en medicina. Profesor de medicina legal y toxicología. Facultad de Medicina y Ciencias de la Salud. Universidad "Rovira i Virgili". 


\section{INTRODUCCIÓN:}

En la primera parte del artículo sobre el "síndrome del latigazo cervical" (SLC) he revisado la incidencia del síndrome, los mecanismos de la lesión, la variedad de lesiones anatómicas que puede causar esas sacudidas y el escaso rendimiento que tienen tanto las radiografías como las TAC o las RM para detectar esas lesiones en la mayor parte de los casos. En esta segunda parte abordo otros aspectos del problema: propongo un cuestionario para facilitar la evaluación clínica de los lesionados, discuto la importancia de las compensaciones económicas en la evolución y las secuelas del cuadro, recojo los datos sobre porcentaje de lesionados curados, porcentaje de reincorporados al trabajo y porcentaje de lesionados con secuelas. Evalúo los distintos factores pronósticos de la recuperación funcional y selecciono los que parecen ser más útiles y termino la revisión con algunos comentarios sobre las terapias disponibles para curar o aliviar el SLC.

\section{EL DIAGNÓSTICO CLÍNICO DEL SÍNDROME DEL LATIGAZO CERVICAL:}

El escaso rendimiento diagnóstico de las pruebas complementarias de imagen obliga a que el diagnóstico y el seguimiento del síndrome del latigazo cervical sean clínicos, usando la anamnesis y la exploración física del enfermo (como siempre, por otro lado). Con el fin de facilitar la recogida de los datos más relevantes para el seguimiento clínico, en la Tabla I propongo un cuestionario para la evaluación de los pacientes. Ha sido confeccionado a partir de la propuesta de Lucena et al. [l].

Tiene dos ventajas, a mi modo de ver: permite realizar y registrar la entrevista médica en diez minutos y facilita la puesta en marcha de investigaciones en colaboración, a menudo imprescindibles para alcanzar un tamaño muestral suficiente.

Las manifestaciones del SLC no son inmediatas. En una investigación prospectiva [2], el 80 \% de los lesionados acudieron a la consulta médica el mismo día de la colisión y el 17,5\% antes de las 48 horas, pero el 2,5\% tardaron más de 48 horas después del choque.

Las manifestaciones más comunes son el dolor del cuello, la cefalea y la restricción de movimientos de la columna cervical, pero también pueden apreciarse disestesias, debilidad, dolor de espalda, cansancio, disfasia, trastornos visuales y dolor temporomandibular.

En la exploración física han de buscarse comportamientos indicativos del dolor del paciente, como los gestos, la fricción de la zona o su protección. Debe anotarse qué movimientos del cuello le causan dolor, cefalea o mareo. La medición de la movilidad del cuello sirve también para valorar la evolución de la lesión y, en caso necesario, para valorar sus secuelas. Las disestesias pueden hacer aconsejables las exploraciones neurológicas específicas.

El dolor del cuello puede clasificarse en varios grados, según su frecuencia y su intensidad (Tabla 2)[2]. El dolor leve sería una molestia, el dolor moderado afectaría las actividades o el trabajo del individuo y el dolor grave las dificultaría muchísimo. Esta clasificación podría usarse también para señalar el alta clínica del afectado.

La constelación de trastornos puede clasificarse en cinco niveles (Tabla 3) [2], en una agrupación con valor predictivo, dado que el riesgo de seguir sufriendo un SLC a los seis meses del accidente aumenta con el grado asignado al lesionado.

El número y la intensidad de las distintas quejas suelen mostrar una buena correlación entre ellas:

- La intensidad del dolor con la presencia de manifestaciones neurológicas del latigazo cervical [3].

- Las cefaleas previas con las posteriores al latigazo cervical [4], siempre que exista también dolor en el cuello, un signo importante de lesión de la columna cervical.

- La cefalea y el dolor en el cuello con la restricción de la movilidad de este [5]. 
Proporcionalmente, la flexión y la extensión son los movimientos más restringidos [6]. Midiendo la amplitud de sus movimientos cervicales, al $80 \%$ de las personas se las pudo diagnosticar correctamente como afectadas por el SLC crónico o como normales (sensibilidad de la prueba 72 \%; especificidad, 88 \%). La capacidad diagnóstica de la exploración se incrementaba al 90 \% incorporando al algoritmo la edad y el sexo del individuo.

- Algunos enfermos, especialmente los afectados crónicos, padecen alteraciones psiquiátricas y trastornos de relación, incluyendo el negativismo y la litigiosidad.

\section{¿DESAPARECERÍA EL PROBLEMA SI NO EXISTIERAN COMPENSACIONES ECONÓMICAS?:}

El hecho de que el SLC sea consecuencia de un accidente de tráfico y que esta situación esté protegida por un seguro lleva a mucha gente a la sospecha, más o menos genérica o basada en anécdotas, de que el afectado pueda exagerar o directamente simular sus lesiones, con el fin de obtener mayores compensaciones económicas o laborales. Es una sospecha que enturbia cualquier problema médico protegido por un seguro, como las enfermedades comunes, los accidentes laborales o las enfermedades profesionales. Todos recordamos anécdotas en ese sentido.

Así, algunos facultativos, tanto clínicos como forenses, creen que la gravedad del SLC está motivada por la reclamación de indemnizaciones económicas y señalan las publicaciones que concluyen que al restringir el sistema de compensación se reducen las demandas $[7,8,9,10,1$ I $]$.

Sin embargo, muchos de los artículos que refutan la existencia o la importancia del SLC tienen limitaciones metodológicas o científicas notables [12]. Tal vez el artículo más citado sea el de Cassidy et al. en el New England Journal of Medicine [9], contestado con varias cartas de los lectores $[13,14,15,16]$. Una de ellas cita la relación entre cobertura sanitaria y duración de los tratamientos: cuando las aseguradoras estadounidenses rehusaron pagar el ingreso hospitalario puerperal más allá del primer día posparto, los días de estancia hospitalaria y los costos de esos cuidados se redujeron enormemente [15]. En otro ejemplo, las reclamaciones a las compañías de seguros en algunas provincias de Australia disminuyeron después de introducir en la legislación barreras burocráticas y dificultades y de encarecer las solicitudes de reclamación.

En una investigación prospectiva realizada en Canadá [2], donde apenas se conceden compensaciones económicas judiciales por estas lesiones, de los 380 pacientes que habían sufrido un SLC, consiguieron evaluar a 126 veinticuatro meses después. A pesar de los tratamientos que hubieran seguido, 44 lesionados padecían aún los trastornos del síndrome (los dolores de los grados 3, 4 ó 5 de la Tabla 2). Esta cifra representa casi un 12 \% de los que habían sufrido la colisión.

En definitiva, pues, no está probado que de no mediar compensaciones económicas desaparecería el SLC.

\section{¿CUÁNTO TARDA EN CURAR EL SLC?:}

La mayoría de afectados por el SLC se recuperan en unas semanas y pueden volver a su trabajo o a sus actividades previas. Sin embargo, a otras el dolor y la incapacidad les duran más de seis meses.

\section{Curación}

Veamos los datos concretos disponibles en la bibliografía cientíica:

- Porcentaje de lesionados curados en menos de una semana: $22 \%$ [17].

- Mediana de tiempo de curación: 31 días [17] y 32 días [18]. Ambos trabajos definieron curación como el momento en el que la aseguradora dejaba de pagar la indemnización por incapacidad. 
- Porcentaje de lesionados con trastornos seis meses después del accidente: $12 \%$ [18], $27 \%$ [19], $35 \%$ [20]. Entre el 19 y el $60 \%$ refieren dolor e incapacidad [21,22].

- Porcentaje de lesionados con persistencia de quejas varios años después del accidente: $18 \%$ [23]. Del 4 al $42 \%[19,24,25,26]$.

Podemos apreciar que el porcentaje de curados crece primero deprisa y después mucho más lentamente. Como los datos provienen de fuentes distintas (de las agrupaciones de aseguradoras, de enfermos que acuden a urgencias de un hospital, de enfermos referidos al hospital desde atención primaria, etc.) debemos combinarlos con precaución. Aún así, digamos que aproximadamente un $20 \%$ de los afectados por un SLC estará curado en una semana, el $50 \%$ en un mes, el $70 \%$ en 6 meses y el $80 \%$ en dos años.

\section{Reincorporación al trabajo}

- Porcentaje de lesionados con incapacidad laboral parcial o completa seis meses después del accidente: El 7 [19], el 9 [27] o el $26 \%$ [28].

- Porcentaje que padecen incapacidad laboral parcial o completa un año después: El 7,8 \% [29]. El $32 \%$ [30].

- En un estudio de médicos forenses españoles [3।], el $45 \%$ de los 145 lesionados estuvieron más de 40 días incapacitados para desempeñar su trabajo o sus tareas habituales (días impeditivos, según el término legal), el 13,8\%, más de 90 días y el 3,4\%, más de 200. No sabemos cuántos días necesitaron los lesionados hasta la sanidad, porque los autores facilitan por separado los días no impeditivos, sin agruparlos con los impeditivos.

\section{¿Cuántos de estos lesionados llegan a la estabilidad con secuelas?}

Hemos de buscar investigaciones españolas porque los estudios foráneos no abordan directamente esta cuestión. En el estudio de los médicos forenses citado [3 I] el 42 \% de los I 45 lesionados padecían secuelas al final del proceso, lo cual parece un porcentaje muy alto. Una razón puede ser que se les diera la sanidad demasiado pronto, de modo que algunos de los afectados que en otros países se clasifican como SLC crónico, sin rendirse en el esfuerzo terapéutico, sean clasificados como "estables con secuelas" en el sistema de valoración médica de las lesiones por accidente de tráfico. El 4 I \% de los lesionados con secuelas (25 de I 45 lesionados) se incluyeron en el apartado de 'cervicalgia postraumática' de dicho baremo [3।].

\section{¿POR QUÉ ALGUNAS PERSONAS TARDAN TANTO EN RECUPERARSE?:}

La explicación más simple es que se trata de otra manifestación de la regla biológica de la dispersión de los valores normales: frente a un problema dado, algunas personas se curan rápidamente y otras necesitan mucho tiempo.

Sin embargo, sería muy útil conocer los datos que nos permitieran seleccionar a los pacientes con mayor riesgo de trastornos persistentes. Algunos sugieren un trastorno a la vez físico y psíquico [32]. Otros señalan que el dolor en el cuello es un fenómeno común en la población general, lo que interactuaría con las lesiones traumáticas [33].

\section{Factores biológicos conocidos de mal pronóstico}

- Dolor en el cuello antes del accidente, sexo femenino y grados 2 y 3 del SLC [30].

- Restricción de la movilidad cervical poco después del accidente. La intensidad del dolor y la presencia de otras manifestaciones del SLC mejoraban la eficacia pronóstica [29]. 
- Restricción de la movilidad cervical a los tres meses del accidente [32].

- La edad avanzada, tener la cabeza inclinada o rotada en el momento del impacto, antecedentes de cefaleas, dolores iniciales más intensos en el cuello y en la cabeza, padecer más manifestaciones del SLC, especialmente de déficit radiculares y tener artrosis más grave en las radiografías [23].

- Restricción de la movilidad cervical poco después del accidente, la intensidad inicial del dolor en el cuello, la intensidad inicial de la cefalea, los antecedentes de cefalea, los antecedentes de traumatismos craneales, la edad avanzada [34].

- Mayor edad, mayor intensidad del dolor del cuello y del dolor de espalda, visión borrosa y menor afectación por mareos [4].

- El sexo femenino y la edad avanzada [17].

- El sexo femenino, la edad avanzada, el dolor a la palpación del cuello, el dolor muscular, el dolor o las parestesias irradiados desde el cuello a los hombros, a los brazos o a las manos y la cefalea. Una mujer de 60 años o más que tuviera todos estos factores de riesgo tardaría una mediana de 262 días en curar, mientras que un hombre de 20 que no los padeciera tardaría 17 días [18].

- La edad avanzada, el número de quejas físicas iniciales y la presentación precoz de los síntomas siguientes: dolor en la parte alta de la espalda, hormigueo o debilidad en las extremidades superiores o trastornos visuales [20].

- Un canal medular de menor calibre [35].

\section{Factores psíquicos de mal pronóstico}

- Puntuar más en las pruebas de afectación del bienestar y menos en las pruebas de tareas de atención y estar más preocupados sobre la incapacidad y el sufrimiento crónicos [23].

- Falta de alivio de los dolores del cuello o de la cabeza con el tiempo. Entre curados y no curados había diferencias significativas en las escalas de bienestar y en la de nerviosismo, pero no la había en la escala de depresión, lo que podría indicar una base somática de los cambios psíquicos. Tampoco hallaron diferencias entre los grupos, ni desviaciones de los valores normales, en las escalas de extraversión o de neuroticismo [36].

- Las puntuaciones de las pruebas de nerviosismo, neuroticismo y concentración [34]. Los autores concluyeron que los problemas psíquicos de los afectados podían ser una consecuencia de los trastornos somáticos del SLC, más que su causa.

- La percepción de la eficacia propia en los primeros días tras el accidente (su confianza en la capacidad de realizar las actividades cotidianas), el sexo y la edad [37].

- La capacidad del enfermo para usar sus recursos para el manejo de situaciones de tensión [38].

- Mayor ansiedad. En cambio, tenían menor sensibilidad al ruido, menos neuroticismo y menos problemas de comportamiento en la infancia. No se apreciaron diferencias significativas respecto a la tensión psicosocial, la afectividad negativa y los rasgos de personalidad [19].

- Más bien problemas psicosociales que físicos, sin diferencias según el sexo del afectado. Posiblemente el uso de ciertas estrategias de afrontamiento era un buen predictor del bienestar psíquico de los lesionados [39]. 


\section{Factores sociales de mal pronóstico}

- Formación académica escasa [30].

- Las reclamaciones legales en curso no influían en la recuperación del paciente [29].

- El entorno social del enfermo [38], con lo que el dolor crónico podría ser una muestra de un trastorno de adaptación psicosocial.

- Tener personas que dependen de uno y no tener trabajo a jornada completa [17].

\section{Factores relacionados con el accidente}

- Viajar en una furgoneta o en un autobús, ser un pasajero del vehículo, chocar contra un objeto en movimiento y que la colisión haya sido frontal o perpendicular [17].

- Tener la cabeza rotada en el momento del impacto. Hasta el $57 \%$ de aquellos con manifestaciones durante más de dos años explican ese antecedente.

En resumen, tenemos multitud de datos, algunos de ellos contradictorios, sobre la importancia de distintos factores pronósticos en la recuperación funcional de los enfermos. Pero una revisión sistemática reciente de los estudios prospectivos sobre el asunto [40] permite calibrar la importancia de cada factor. Después de aplicar sus criterios de selección a los artículos, los investigadores trabajaron sobre 50 publicaciones, en inglés, francés, holandés o alemán. Sus resultados están resumidos en la Tabla 4.

El segundo artículo que nos facilitará la selección de los factores pronósticos más importantes es el de Hartling et al. [20], quienes, en una investigación prospectiva, cuantificaron el riesgo de SLC crónico derivado de distintos factores. Además, desarrollaron un algoritmo para seleccionar a los lesionados con riesgo más alto de padecer un SLC crónico (Figura I). Es un algoritmo sencillo y razonablemente eficaz: según el artículo, tiene una sensibilidad del $91,5 \%$ y una especificidad del $5 \mathrm{I}, 4 \%$. Los lesionados clasificados como 'de riesgo alto' tienen un 50,7 \% de probabilidades de padecer un SLC crónico y los 'de riesgo bajo', un 91,7\% de probabilidades de no padecerlo (valores predictivos positivo y negativo, respectivamente).

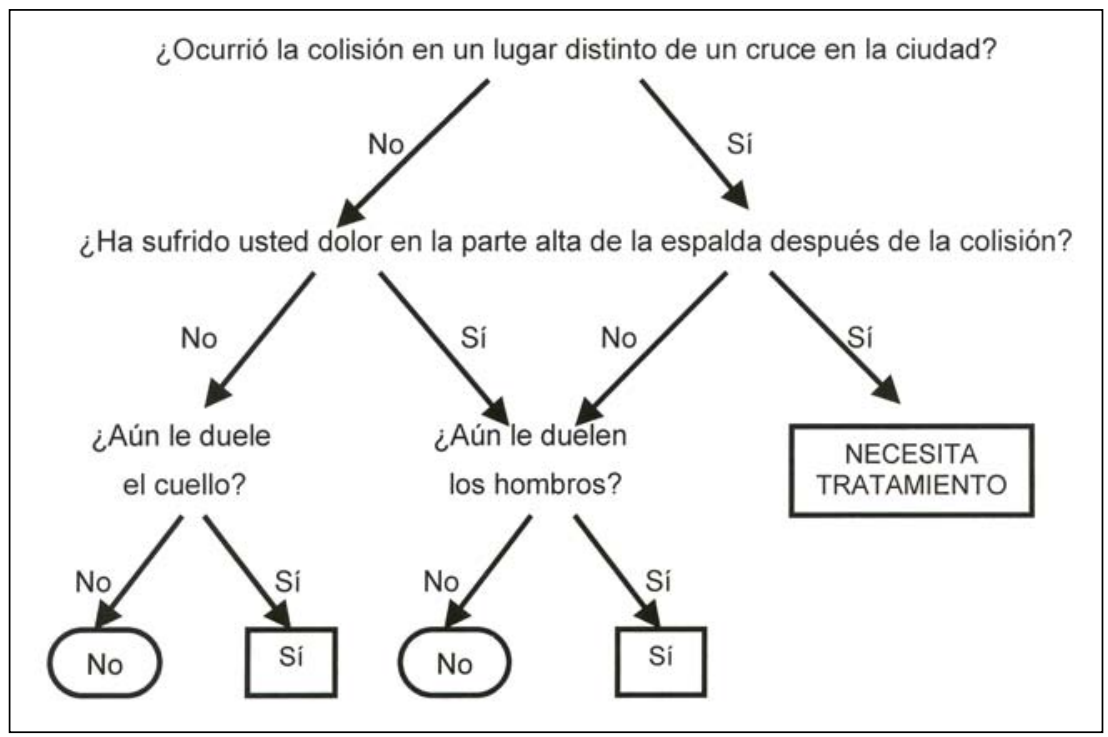

Figura 1.- Algoritmo para identificar a los lesionados con mayor riesgo de padecer un SLC crónico después de un alcance entre vehículos [20]. 


\section{El 'modelo biológico de lesión'}

Simplificando, "el resultado para un paciente individualizado puede seguir dos caminos: el dolor se resolverá en los primeros meses, o bien persistirá indefinidamente." [4I]. La duración del dolor y la incapacidad dependen del órgano lesionado por el traumatismo y de su capacidad de recuperación. Si la tiene, el enfermo podrá recuperarse en semanas o pocos meses. Si se han afectado los discos, las articulaciones interapofisarias o las cápsulas articulares, es muy probable que el lesionado sufra dolor e incapacidad crónicos.

Lamentablemente, aún no podemos concretar con pruebas de imagen cuál es el órgano lesionado. Si a esa dificultad le añadimos la variabilidad en el pronóstico y los factores predictores de tipo psíquico, es comprensible que algunos traumatólogos o reumatólogos vean a algunos de sus pacientes como histéricos o hipocondríacos. Si además el lesionado puede recibir una indemnización por los perjuicios sufridos, el médico fácilmente sospechará que este sea un simulador.

\section{OPCIONES TERAPÉUTICAS FRENTE AL LATIGAZO CERVICAL:}

Están descritas con detalle en algunos artículos recientes [42,43] y el objetivo de este artículo no es revisarlas. Sin embargo, interesa mencionar los aspectos relacionados más directamente con la evaluación forense de las lesiones sufridas.

- Se han propuesto multitud de opciones terapéuticas para resolver este problema, lo que no es extraño, dado que no lo comprendemos a fondo. La utilidad de las terapias no siempre ha sido validada científicamente.

- El reposo y el collarín blando continúa siendo una prescripción frecuente en España para el latigazo cervical [3।]. Sin embargo, desde la segunda mitad de los años ochenta se cuestiona su utilidad, porque los estudios clínicos llegaron a la conclusión de que la movilización precoz era mucho más eficaz $[44,45,46]$.

- Ejercicios en casa o fisioterapia ambulatoria. Un ensayo clínico prospectivo, aleatorizado y a ciegas [45] no encontró diferencias significativas entre los resultados de ambas pautas. Sin embargo, ambas fueron superiores al collarín más analgesia. Otro estudio aleatorizado [47] concluía que para el SLC agudo era suficiente un programa de ejercicios para realizar en casa (que incluía mejora de la movilidad del cuello y de los hombros, relajación y consejos generales), siempre que se siguiera diariamente.

- El modelo biopsicosocial del SLC [48] propone un equipo multidisciplinar para la rehabilitación [42]. Sin embargo, no parecen haber pruebas científicas de que ese abordaje sea más efectivo que otros métodos rehabilitadores [43].

Sea cual sea la opción terapéutica seleccionada, incluso los enfermos que llevan años con incapacidad laboral ven disminuir su dolor y aumentar la tolerancia al dolor y la sensación de bienestar si reciben los tratamientos médicos y psicológicos adecuados [22,49].

Las conclusiones más prácticas de esta segunda parte de la revisión están recogidas en la Tabla 5. 
TABLA I:

\section{HOJA DE RECOGIDA DE DATOS PARA LA VALORACIÓN DEL PACIENTE CON SÍNDROME DEL LATIGAZO CERVICAL}

\section{A. Antecedentes personales:}

Nombre y apellidos:

Edad: Sexo: Peso: ___ $\mathrm{kg}$ Profesión:

Actividad laboral: No/T. parcial / T. completo:

Antecedentes patológicos:

Cefaleas Braquialgias Parestesias EESS Vértigos Acúfenos Artrosis cervical Artrosis en otro(s) lugar(es) Problemas psiquicos Problemas sociales

Especificar.

\section{B. Características del cuadro:}

Fecha del traumatismo: / / Fecha del primer examen médico: I / Tipo de suceso: Agresión / Otros:

Accidente de tráfico:

- Tipo de vehículo: coche moto autobús tren

- Tipo de accidente: choque frontal choque lateral choque posterior frenazo brusco otros:

- Velocidad de los vehículos: lesionado: $\mathrm{km} / \mathrm{h} \quad$ El otro: $\mathrm{km} / \mathrm{h}$

- Posición del lesionado: conductor (cinturón de seguridad: sí / no) acompañante (cinturón de seguridad: si / no) asiento trasero (cinturón de seguridad: sí / no)

- Posición de la cabeza en el momento del choque:

Aparición de los trastornos a las: $1-6 \mathrm{~h} \quad 6-12 \mathrm{~h} \quad 12-24 \mathrm{~h} \quad 24-48 \mathrm{~h} \quad>48 \mathrm{~h}$

Asistencia médica a las: $\quad 1-6 \mathrm{~h} \quad 6-12 \mathrm{~h} \quad 12-24 \mathrm{~h} \quad 24-48 \mathrm{~h} \quad 48 \mathrm{~h}$

Exploraciones médicas iniciales practicadas:

Exploración física Radiografias cervicales TAC RM Otros (especificar):

Resultados:

- Otras lesiones en el accidente: No / Si: 


\section{TABLA I CONTINUACIÓN:}

C. Manifestaciones del SLC (cuantificar la gravedad con cruces):

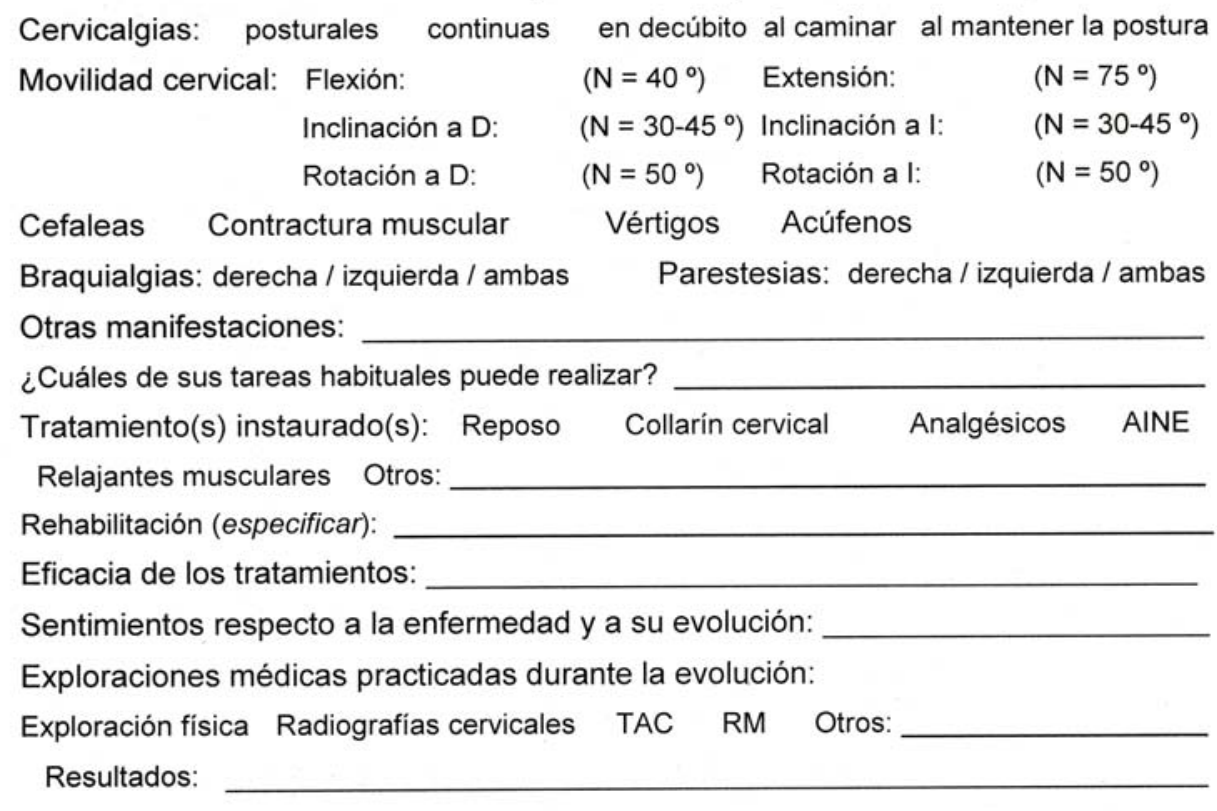

\section{Valoración medicoforense:}

Días hasta curar: Días incapacidad para la ocupación habitual: ¿Alguna concausa alargó el proceso? No Si (especificar): SECUELAS:

Cervicalgias: posturales continuas en decúbito

\begin{tabular}{|c|c|c|c|c|c|}
\hline \multirow{3}{*}{\multicolumn{2}{|c|}{ Movilidad cervical: }} & : Flexión: & $\left(\mathrm{N}=40^{\circ}\right)$ & Extensión: & $\left(\mathrm{N}=75^{\circ}\right)$ \\
\hline & & Inclinación a D: & $\left(\mathrm{N}=30-45^{\circ}\right)$ & Inclinación a I: & $\left(\mathrm{N}=30-45^{\circ}\right)$ \\
\hline & & Rotación a D: & $\left(\mathrm{N}=50^{\circ}\right)$ & Rotación a I: & $\left(\mathrm{N}=50^{\circ}\right)$ \\
\hline Cefaleas & Cont & tractura muscular & Vértigos & Acúfen & \\
\hline Braquialgias: & & izquierda & ambas & (cuantificar) & \\
\hline Parestesias: & & izquierda & ambas & (cuantificar) & \\
\hline
\end{tabular}

Otras manifestaciones (especificar):

Necesidad de incapacidad permanente: No Sí:

Parcial Total Absoluta




\begin{tabular}{|c|c|c|c|c|}
\hline \multirow{2}{*}{$\begin{array}{c}\text { FRECUENCIA DEL } \\
\text { DOLOR }\end{array}$} & \multicolumn{4}{|c|}{ INTENSIDAD DEL DOLOR } \\
\cline { 2 - 5 } & SIN DOLOR & LEVE & MODERADO & GRAVE \\
\hline Nunca & 0 & 0 & 0 & 0 \\
\hline Esporádico & 0 & 1 & 2 & 3 \\
\hline Habitual & 0 & 2 & 3 & 4 \\
\hline Diario & 0 & 2 & 4 & 5 \\
\hline
\end{tabular}

Tabla 2.- Gradación del dolor en el síndrome del latigazo cervical [2]. Los incluidos en los grados 0,1 ó 2 podrían ser excluidos del diagnóstico de sindrome del latigazo cervical.

\begin{tabular}{|c|c|}
\hline GRADO & PRESENTACIÓN CLINICA \\
\hline 0 & No hay síntomas del cuello ni signos físicos. \\
\hline 1 & $\begin{array}{l}\text { Sintomas del cuello (dolor, rigidez o dolor con la palpación) sin signos } \\
\text { físicos. }\end{array}$ \\
\hline \multirow{3}{*}{2} & Sintomas del cuello y signos musculoesqueléticos. \\
\hline & 2a $\quad$ Dolor en algunos puntos, movilidad cervical normal \\
\hline & 2b 1 Dolor en algunos puntos; movilidad cervical restringida \\
\hline 3 & $\begin{array}{l}\text { Sintomas del cuello y signos neurológicos: disminución o ausencia } \\
\text { de los reflejos tendinosos profundos, debilidad y déficit sensitivos. }\end{array}$ \\
\hline 4 & Síntomas del cuello y fractura o luxación cervical. \\
\hline
\end{tabular}

Tabla 3.- Clasificación de Québec de la gravedad de los trastornos asociados al latigazo cervical [2].

\begin{tabular}{|l|c|}
\hline \multicolumn{1}{|c|}{ FACTORES PRONÓSTICOS } & CALIDAD \\
\hline Dolor inicial intenso & Factor adverso importante \\
\hline $\begin{array}{l}\text { Restricción de la movilidad del cuello } \\
\text { Gran número de trastornos } \\
\text { Problemas psiquicos previos } \\
\text { Nerviosismo } \\
\text { Accidente en la autopista } \\
\text { Necesidad de la fisioterapia }\end{array}$ & \\
\hline $\begin{array}{l}\text { Edad avanzada } \\
\text { Sexo femenino } \\
\text { Respuesta psicológica aguda intensa } \\
\text { Cifosis segmentaria del cuello } \\
\text { Colisión desde atrás }\end{array}$ & \\
Indemnización económica & factores adversos \\
\hline
\end{tabular}

Tabla 4.- Factores pronósticos más fiables para el SLC [40]. 
El escaso rendimiento diagnóstico de las radiografías, las TAC y las RMN obligan a que el diagnóstico y el seguimiento del síndrome del latigazo cervical sean clínicos, usando la anamnesis y la exploración física del enfermo.

- El uso de un cuestionario común facilitaría la evalución clínica de los lesionados y tendría ventajas científicas.

- La amplitud de los movimientos cervicales es una buena prueba diagnóstica de SLC crónico.

- A pesar de que pueda ser una creencia común, no está probado que de no mediar compensaciones económicas desaparecería el SLC.

- En cifras aproximadas, un $20 \%$ de los afectados por un SLC estará curado en una semana, el $50 \%$ en un mes, el $70 \%$ en 6 meses y el $80 \%$ en dos años.

- En España, cerca del $40 \%$ de los lesionados por el SLC quedan clasificados como "estables con secuelas". Tal vez se les da la sanidad demasiado pronto en el sistema de valoración médica de las lesiones por accidente de tráfico.

- Tenemos multitud de datos, algunos de ellos contradictorios, sobre la importancia de distintos factores pronósticos en la recuperación funcional de los enfermos. Los factores más importantes, según una revisión sistemática reciente, están resumidos en la Tabla 4.

El pronóstico del SLC depende de cuál haya sido el órgano lesionado por el traumatismo. Sin embargo, las pruebas de imagen no nos permiten concretar cuál es. Esa inseguridad puede explicar en parte por qué a muchos de esos lesionados se les vive como hipocondríacos o directamente como simuladores.

Tabla 5.- Deducciones prácticas de esta segunda parte de la revisión.

\section{BIBLIOGRAFÍA:}

I. Lucena J, Subirana M, Jiménez A. Protocolo de valoración médicoforense en el síndrome del latigazo cervical. En: Libro de las Segundas jornadas catalanas de actualización en medicina forense. Barcelona, noviembre de 1993.

2. Hartling L, Brison RJ, Ardern C, Pickett W. Prognostic Value of the Quebec Classification of Whiplash-associated Disorders. Spine 2001;26:36-4I.

3. Kasch H, Bach FW, Stengaard-Pedersen K, Jensen TS. Development in pain and neurologic complaints after whiplash: A I-year prospective study. Neurology 2003; 60:743-749.

4. Radanov BP, Sturzenegger M, Di Stefano G, Schnidrig A, Aljinovic $M$. Factors influencing recovery from headache after common whiplash. BMJ 1993;307: 652-655.
5. Kasch H, Stengaard-Pedersen K, Arendt-Nielsen L, Jensen TS. Headache, Neck Pain, and Neck Mobility After Acute Whiplash Injury. Spine 2001;26:1246-I25I.

6. Dall'Alba PT, SterlingMM, Treleaven JM, Edwars SL, Jull GA. Cervical Range of Motion Discriminates Between Asymptomatic Persons and Those With Whiplash. Spine 200I;26:2090-2094.

7. Schrader H, Obelieniene D, Bovim G, Surkiene D, Mickeviciene D, Miseviciene I, Sand T. Natural evolution of late whiplash syndrome outside the medicolegal context. Lancet 1996;347:1207-I2II.

8. Obelienne D, Schrader H, Bovim G, et al. Pain after whiplash: A prospective controlled inception cohort study. J Neurol Neurosurg Psychiatry 1999;66:279-283. 
9. Cassidy JD, Carroll LJ, Côté P et al. Effect of eliminating compensation for pain and suffering on the outcome of insurance claims for whiplash injury. N Engl J Med 2000; 342:II79-II86.

10. Ferrari R. Editorial: the Many Facets of Whiplash. Spine 2001;26:2063-2064.

II. Ferrari R. Whiplash - review of a commnoly misunderstood injury. Am J Med 2001;162-163 (carta al editor).

12. Freeman MD, Croft AC, Rossignol AM, Weaver DS, Reiser M. A review and methodologic critique of the literature refuting whiplash syndrome. Spine 1999; 24:86-96.

13. Freeman MD, Rossignol AM. Effect of eliminating compensation for pain and suffering on the outcome of insurance claims. N Engl J Med 2000;343: III8-III9 (carta al editor).

14. Merskey H, Teasell RW. Effect of eliminating compensation for pain and suffering on the outcome of insurance claims. N Engl J Med 2000;343:III9 (carta al editor).

15. Clionsky M. Effect of eliminating compensation for pain and suffering on the outcome of insurance claims. N Engl J Med 2000;343:III9 (carta al editor).

16. Russell RS. Effect of eliminating compensation for pain and suffering on the outcome of insurance claims. N Engl J Med 2000;343:III9-II20 (carta al editor).

17. Harder S, Veilleux M, Suissa S. The effect of socio-demographic and crash-related factors on the prognosis of whiplash. J Clin Epidemiol 1998;51:377-384.

18. Suissa S, Harder S, Veilleux M. The relation between initial symptoms and signs and the prognosis of whiplash. Eur Spine J 2001;10:44-49.

19. Radanov BP, Di Stefano G, Schnidrig A, Sturzenegger M. Psychosocial stress, cognitive performance and disability after common whiplash. J Psychosom Res 1993;37:1-10.

20. Hartling L, Pickett W, Brison RJ. Derivation of a clinical decision rule for whiplash associated disorders among individuals involved in rear-end collisions. Accid Anal Prev 2002;34:53I-539.

21. Freeman MD, Croft AC, Rossignol AM. "Whiplash associated disorders: redefining whiplash and its management" by the Quebec Task Force. A critical evaluation. Spine 1998;23:1043-1049.

22. Stovner LJ. The nosologic status of the whiplash syndrome: A critical review based on a methodological approach. Spine 1996;21:2735-2746.

23. Radanov BP, Sturzenegger M, Di Stefano G. Long-term outcome after whiplash injury. A 2-year follow-up considering features of injury mechanism and somatic, radiologic, and psychosocial findings. Medicine (Baltimore) 1995;74:281-297.

24. Barnsley L, Lord S, Bogduk N. Clinical review: whiplash injury. Pain 1994;58: 283-307.

25. Carette S. Whiplash injury and chronic neck pain. N EngI J Med 1994;330: 1083-1084.

26. Deans GT, Magalliard K, Rutherford WH. Neck sprain: a major cause of disability following car accidents. Injury 1987; 84:161-164. 27. Provinciali L, Baroni M. Clinical approach to whiplash injuries:
A review. Phys Rehabil Med 1999;11:339-368.

28. Spitzer WO, Skovron ML, Salmi LR et al. Monograph of the Québec Task force on Whiplash-Associated Disorders: Redefining "whiplash" and its management. Spine 1995;20(Suppl):SI-S73.

29. Kasch H, Bach FW, Jensen TS. Handicap after acute whiplash injury: a I-year prospective study of risk factors. Neurology 2001;56:1637-1643.

30. Sterner Y, Toolanen G, Gerdle B, Hildingsson C. The incidence of whiplash trauma and the effects of different factors on recovery. J Spinal Disord Tech 2003;16:195-199.

3I. Smith E, Mayans J, Montañana JV et al. Esguince cervical postraumático. Valoración lesional y terapéutica. Repercusiones médicas y legales. Rev Soc And Traum Ortop 2001;21:189-194.

32. Gargan M, Bannister G, Main C, Hollis S. The behavioural response to whiplash injury. J Bone Joint Surg Br 1997;79:523-526.

33. Guez M, Hildingsson C, Nilsson M, Toolanen G. The prevalence of neck pain: a population-based study from northern Sweden. Acta Orthop Scand 2002;73: 455-459.

34. Radanov BP, Sturzenegger M. Predicting recovery from common whiplash. Eur Neurol 1996;36:48-5I.

35. Pettersson K, Karrholm J, Toolanen G, Hildingsson C. Decreased width of the spinal canal in patients with chronic symptoms after whiplash injury. Spine 1995;20:1664-1667.

36. Radanov BP, Begre S, Sturzenegger M, Augustiny KF. Course of psychological variables in whiplash injury--a 2-year follow-up with age, gender and education pair-matched patients. Pain 1996;64:429-434.

37. Kyhlback M, Thierfelder T, Soderlund A. Prognostic factors in whiplash-associated disorders. Int I Rehabil Res 2002;25:181-187. 38. Heikkila H, Heikkila E, Eisemann M. Predictive factors for the outcome of a multidisciplinary pain rehabilitation programme on sick-leave and life satisfaction in patients with whiplash trauma and other myofascial pain: a follow-up study. Clin Rehabil 1998;12:487-496.

39. Soderlund A, Lindberg P. Long-term functional and psychological problems in whiplash associated disorders. Int J Rehabil Res 1999;22:77-84.

40. Scholten-Peeters GG, Verhagen AP, Bekkering GE, van der Windt DA, Barnsley L, Oostendorp RA, Hendriks EJ. Prognostic factors of whiplash-associated disorders: a systematic review of prospective cohort studies. Pain 2003;104:303-322.

4I. Combalía A, Suso S, Segur JM, García S, Alemany FX. Síndrome del latigazo cervical. Med Integral 2001;38:95-102.

42. Scholten-Peeters GGM, Bekkering GE, Verhagen AP, et al. Clinical Practice Guideline for the Physiotherapy of Patients With Whiplash-Associated Disorders. Spine 2002;27:412-422.

43. Karjalainen K, Malmivaara A, Van Tulder M et al. Multidisciplinary Biopsychosocial Rehabilitation for Neck and Shoulder Pain Among Working Age Adults. A systematic Review Within the Framework of the Cochrane Collaboration Back Review Group. Spine 2001;26:174-181. 
44. Mealy K, Brennan H, Fenelon GC. Early mobilization of acute whiplash injuries. Br Med J (Clin Res Ed) 1986;292:656-657.

45. McKinney LA, Dornan J0, Ryan M. The role of physiotherapy in the management of acute neck sprains following road-traffic accidents. Arch Emerg Med 1989;6:27-33.

46. Borchegrevink GE, Kaasa A, McDonagh D, et al. Acute treatment of whiplash neck sprain injuries. A randomized trial of treatment during the first 14 days after car accident. Spine 1998;23:25-31.
47. Soderlund A, Olerud C, Lindberg P. Acute whiplash-associated disorders (WAD): the effects of early mobilization and prognostic factors in long-term symptomatology. Clin Rehabil 2000; 14:457-467.

48. Ferrari R. Prevention of chronic pain after whiplash. Emerg Med J 2002;19: 526-530.

49. Olivegren H, Jerkvall N, Hagstrom Y, Carlsson J. The long-term prognosis of whiplash-associated disorders (WAD). Eur Spine J 1999;8(5):366-70. 